\title{
Vapor-Phase Microprinting of Multicolor Phosphorescent Organic Light Emitting Device Arrays
}

\author{
Gregory J. McGraw and Stephen R. Forrest*
}

Three primary factors govern the cost and performance of organic light emitting diode (OLED) displays that are now gaining widespread acceptance in the mobile electronic appliance market. ${ }^{[1]}$ The emitting devices themselves used in the display "frontplane," must be of very high efficiency. For this purpose, nearly $100 \%$ internally efficient small molecule electrophosphorsecent OLEDs (PHOLEDs) ${ }^{[2]}$ are almost universally used for red, and to a lesser extent for green emission. The second factor is the transistor "backplane". Primarily, low temperature polysilicon transistor arrays are used for this purpose, ${ }^{[3]}$ although metal oxide transistors are gaining acceptance. ${ }^{[4]}$ The last factor that forms the "three-legged stool" supporting the widespread use of OLED displays, particularly for the large television and monitor markets, is a low-cost, fast, and highresolution means for pixel patterning. Individual subpixels can range in size from $30 \mu \mathrm{m}$ to $100 \mu \mathrm{m}$, depending on the size and resolution of the display.

One particular challenge to achieving low-cost and rapid patterning of organic electronic devices is that organic materials are often chemically incompatible with traditional photolithographic processing. ${ }^{[5]}$ Hence, electronically active features are often fabricated by methods that combine film growth and patterning into a single step. The most commonly used method is vacuum thermal evaporation using thin metal shadow masks. ${ }^{[6]}$ However, such masks are susceptible to clogging and are difficult to manage over large areas. An alternative is to use a smaller "scanning shadow mask" whose precise position control relative to the mother glass presents significant challenges. ${ }^{[7]}$ Other techniques include solvent-based techniques such as inkjet ${ }^{[8]}$ and dry-transfer including laser induced thermal imaging ${ }^{[9]}$ and molecular jet printing. ${ }^{[10]}$ A practical processing technique must be capable of rapid, high-resolution patterning, and it must also be compatible with high efficiency PHOLED architectures fabricated from low molecular weight materials. Finally, the technique must be scalable to Gen 8 substrates ${ }^{[11]}$ and beyond.

To solve many of these problems, organic vapor jet printing (OVJP) has been introduced ${ }^{[12]}$ to finely pattern small molecule

Dr. G. J. McGraw

Department of Physics and Applied Physics Program University of Michigan

Ann Arbor, MI 48109, USA

Prof. S. R. Forrest

Departments of Electrical Engineering and Computer

Science and Materials Science and Engineering

University of Michigan

Ann Arbor, MI 48109, USA

E-mail: stevefor@umich.edu

DOI: 10.1002/adma.201204410 organic electronic devices, and particularly multicolor PHOLED arrays. In OVJP, evaporated organic material is volatilized by, and subsequently entrained in a heated stream of inert carrier gas. Vapor from multiple sources, such as the host and dopant in a PHOLED light emitting layer (EML), are then mixed in a common fluidic channel. The heated channel distributes the vapor mixture among an array of nozzles, which collimate it into well-defined jets that impinge on a proximally located chilled substrate in a vacuum chamber. The organic vapor condenses into well-defined features on the substrate, and patterns are generated by moving the substrate relative to the nozzle array. ${ }^{[13]}$

High-resolution printing and scalability can be achieved using an array of microfabricated nozzles to print multiple PHOLED segments of different colors simultaneously. Efficient single-color phosphorescent devices with features as small as $16 \mu \mathrm{m}$ have been demonstrated using this method. ${ }^{[14]}$ Growths of multicolor arrays of PHOLED segments have also been achieved, albeit with a single nozzle and comparatively large feature sizes of $1 \mathrm{~mm} \cdot{ }^{[15]}$

Here, we demonstrate that OVJP is an effective method for printing multicolor PHOLED arrays at the scale required in large area display applications. The electroluminescence of printed arrays of red and green segments is characterized both spatially and spectrally to determine feature size, and whether cross-contamination from adjacent printed features is present; that is, whether or not there is "overspray" of dopants between adjacent pixels. Light-emitting features of $100 \mu \mathrm{m}$ width were demonstrated. A sensitive electrophosphorescent probe OLED was used to determine that adjacent red and green subpixels can be printed at separations of $210 \mu \mathrm{m}$ with no significant overspray contamination with the nozzle shapes and experimental conditions used here. We find that a further reduction in the separation between adjacent subpixels to $50 \mu \mathrm{m}$ (corresponding to $150 \mu \mathrm{m}$ full color pixel separation in a red-green-blue pixel display) is possible by using a nozzle-to-substrate separation distance of $g=10 \mu \mathrm{m}$ along with raised barriers integrated onto the substrate surface.

Two different nozzle geometries were fabricated for this study (see Experimental Section). The first nozzle geometry consisted of a $20 \mu \mathrm{m} \times 200 \mu \mathrm{m}$ rectangular aperture at the base of a converging inlet. The outlet aperture of this simpleconverging (S-C) nozzle is as shown in Figure 1a. Nozzles were arranged in two separate, staggered arrays: one to deposit a red host and dopant combination, and the other for a green combination. The ten nozzles in each array had a center-tocenter spacing of $250 \mu \mathrm{m}$. Arrays were offset such that adjacent printed lines were of the alternate color, resulting in an adjacent red-green pixel spacing of $125 \mu \mathrm{m}$. The long dimensions of the nozzles are parallel to the direction of substrate motion. 


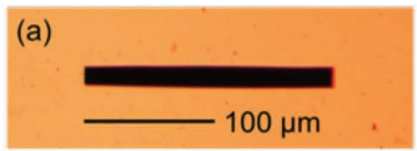

(c) $\begin{array}{llll}0 & 100 & 200 & 300 \\ \end{array}$
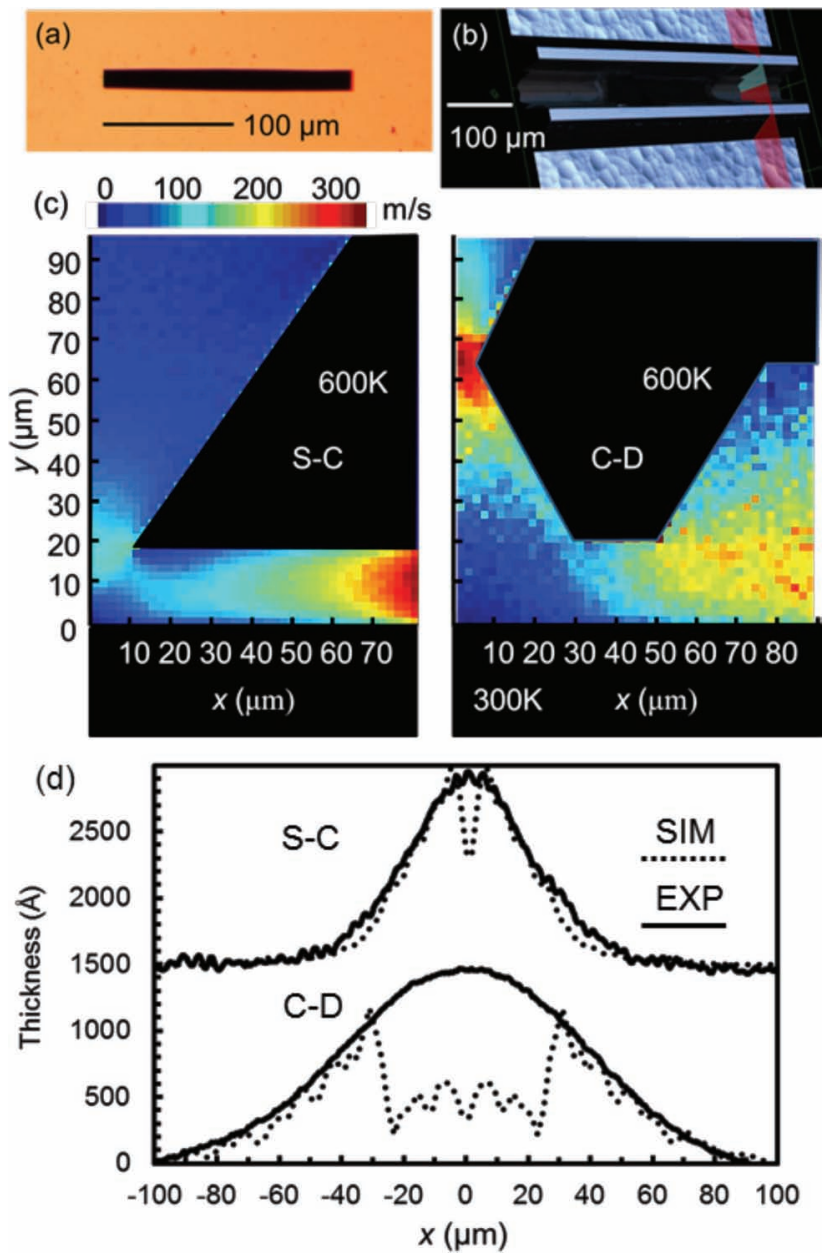

Figure 1. a) Micrograph of the aperture of a simple-converging (S-C) nozzle. b) A rendering of the outlet of a converging-diverging (C-D) nozzle obtained by optical profilometry. c) Simulated velocity fields of carrier gas through cross sections for S-C (left) and converging-diverging (right) nozzles. Both nozzles are symmetric around their left boundaries. Gas flows from the top of the nozzle to the substrate at the bottom, and then out through the right boundary. d) Thickness profiles of printed organic features as a function of distance from the nozzle centerline for both nozzle geometries. Solid lines indicate stylus profilometry measurements. Profiles predicted by simulations are shown using dotted lines.

This array prints high-resolution features, whose thickness profiles are approximately Gaussian. ${ }^{[14]}$ Hence, a line printed in a single pass may have an intensity variation across the pixel under electrical pumping. Multiple passes of the nozzle array are, therefore, preferable to form mesa-like features that result in uniform emission across the pixel width.

The converging-diverging (C-D) nozzle geometry consists of a converging inlet along with a diverging outlet, as shown in Figure 1b. The diverging section creates a diffuse flow that results in a more mesa-like deposition profile. The outlet of the nozzle is $70 \mu \mathrm{m} \times 500 \mu \mathrm{m}$, and its throat is $20 \mu \mathrm{m} \times$ $200 \mu \mathrm{m}$. Two staggered arrays of five C-D nozzles, one for each color, were fabricated. Nozzles in each array were on a $1 \mathrm{~mm}$ center-to-center spacing. While C-D nozzles are often used to accelerate jets to supersonic velocity, ${ }^{[16]}$ the proximity of the substrate prevents such acceleration in this case.

Simulated carrier gas velocity fields for the two nozzle geometries are shown in Figure 1c. Both nozzle geometries are modeled normal to their long dimension using a 2 dimensional direct simulation Monte Carlo (DSMC) method.[17] Gas flow enters through the top of the simulated volume at a pressure of $3500 \mathrm{~Pa}$, and leaves along the right boundary. The left boundary is a symmetry plane. The nozzle temperature is $600 \mathrm{~K}$ and the substrate is at $300 \mathrm{~K}$. The details of this model are discussed further in the Experimental Section. It is apparent that the gas flow immediately beneath the S-C nozzle aperture is at a relatively low $\left(<100 \mathrm{~m} \mathrm{~s}^{-1}\right)$ velocity, accelerating as it moves along the lateral $(\hat{x})$ direction towards the outlet. The gas leaves the nozzle aperture with higher velocity in the C-D nozzle and then further accelerates as it moves beyond the protruding lower surface of the C-D nozzle.

These two flow patterns influence the deposit shape as calculated in Figure 1d. Both modeling and experiment (also shown) suggest that the S-C nozzle brings organic vapor into close proximity with the substrate in a region of relatively stagnant carrier gas flow, allowing the vapor to adsorb onto the substrate without significant convective broadening. The acceleration of carrier gas as it approaches the right-hand model boundary does not substantially increase the width of the printed features, since virtually all of the organic molecules are adsorbed close to the nozzle centerline. The C-D nozzle, however, directs organic vapor outward along the diverging slope of the nozzle wall, imparting it with substantial velocity along $\hat{x}$. While most organic material adsorbs on the substrate within the stricture it forms with the nozzle, the outer slope of the C-D nozzle produces an expanding jet that further accelerates the remaining organic vapor. Consequently, the C-D nozzle prints features with diffuse edges compared to the S-C nozzle. Note that there is agreement between the measured feature width for both S-C and C-D nozzles, and the widths of features for these nozzle geometries predicted by the DSMC model using previously described boundary conditions. Growth conditions for printed features are the same as for the green emitting film in the Experimental Section, however substrate speed was slowed to $2 \mathrm{~mm} \mathrm{~s}^{-1}$. This yielded thicker $(\approx 100 \mathrm{~nm})$ features that could be readily characterized by stylus profilometry. Features were printed at nozzle aperture-to-substrate distance $g=20 \mu \mathrm{m}$. The DSMC model, however, predicts that deposited films using C-D nozzles have a decreased thickness near the center of the pixel, which is not observed experimentally. This is most likely due to an underestimation of diffusive surface transport of organic material by the model.

The nozzle array deposits multiple features in parallel in a single pass, resulting in uniformity in both feature size and thickness. Printed lines of 4,4'-bis(N-carbazolyl)-1,1'-biphenyl (CBP) doped with tris(2-phenylpyridinato) iridium(III) (Irppy $\left.{ }_{3}\right)$ deposited onto a Si wafer by an array of ten SC nozzles at $g=$ $10 \mu \mathrm{m}$ are shown in Figure 2a. The thickness of printed features is $310 \pm 20 \AA$, which is consistent with the emissive region thickness in a conventional PHOLED. Variation in the thickness and width across an array of printed features measured by stylus profilometry is less than $10 \%$ as shown in Figure $2 \mathrm{~b}$. The printed feature full width at half maximum (FWHM) is 

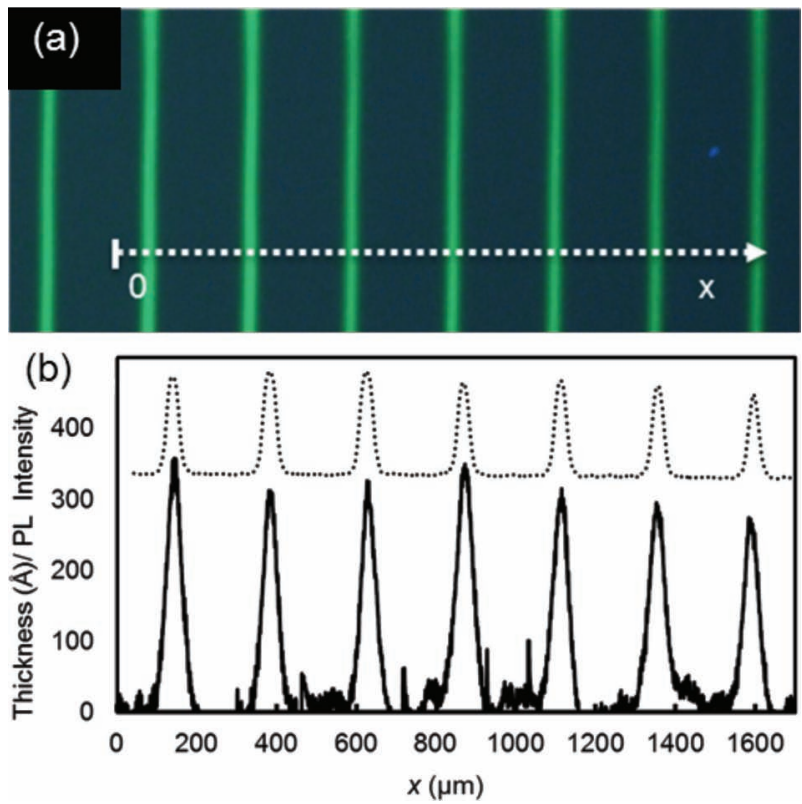

Figure 2. a) Photoluminescence of lines of $\operatorname{Ir}(\mathrm{ppy})_{3}$ doped $C B P$ thin films deposited on an Si substrate by an array of S-C nozzles at a nozzle-tosubstrate gap of $g=10 \mu \mathrm{m}$. Photoluminescence is excited by ultraviolet illumination. b) Film-thickness profiles measured by stylus profilometry (solid line) perpendicular to the printed line longitudinal axes. The size of the features was also measured by the photolumienscence intensity (dotted line)

$42 \pm 4 \mu \mathrm{m}$, consistent with the feature size of $35 \pm 4 \mu \mathrm{m}$ obtained from the photoluminescence intensity profile.

Table 1 lists the widths at $50 \%$ and $10 \%$ of maximum intensity for an array of electrophosphorescent devices printed using S-C nozzles at $g=20$, and C-D nozzles at $g=20,50$, and $100 \mu \mathrm{m}$. The red and green emitting features are narrowest and have well defined edges for $g=20 \mu \mathrm{m}$. In contrast, the emissive features are larger and have diffuse edges at $g=100 \mu \mathrm{m}$. Figure 3a shows spectrally resolved intensity scans taken perpendicular to the printed lines at several $g$ values. The electroluminescence intensity across the width of both red and green printed features is roughly Gaussian, as shown in the line scan in Figure 3b. The general trend of broader features surrounded by more diffuse boundaries with increasing $g$ is apparent. For a given set of conditions, the red and green pixels have a similar FWHM.

Table 1. Widths of printed OLED pixels at their full-width half-maximum (FWHM) brightness and $10 \%$ of maximum (10\%M) brightness.

\begin{tabular}{lcccc}
\hline $\begin{array}{l}\mathrm{g}^{\mathrm{a})} \\
{[\mu \mathrm{m}]}\end{array}$ & $\begin{array}{c}\left.\mathrm{C}^{\mathrm{b}}\right) \text { FWHM } \\
{[\mu \mathrm{m}]}\end{array}$ & $\begin{array}{c}\mathrm{C}^{\mathrm{b})} 10 \% \mathrm{M} \\
{[\mu \mathrm{m}]}\end{array}$ & $\begin{array}{c}\left.\mathrm{R}^{\mathrm{b}}\right) \mathrm{FWHM} \\
{[\mu \mathrm{m}]}\end{array}$ & $\begin{array}{c}\mathrm{R}^{\mathrm{b})} 10 \% \mathrm{M} \\
{[\mu \mathrm{m}]}\end{array}$ \\
\hline $20(\mathrm{~S}-\mathrm{C})^{\mathrm{c})}$ & $94 \pm 18$ & $124 \pm 18$ & - & - \\
$20(\mathrm{C}-\mathrm{D})^{\mathrm{c})}$ & $97 \pm 22$ & $166 \pm 40$ & $127 \pm 13$ & $250 \pm 45$ \\
$50(\mathrm{C}-\mathrm{D})^{\mathrm{c})}$ & $200 \pm 59$ & $275 \pm 76$ & $214 \pm 12$ & $365 \pm 7$ \\
$100(\mathrm{C}-\mathrm{D})^{\mathrm{c})}$ & $263 \pm 47$ & $408 \pm 31$ & $299 \pm 21$ & $550 \pm 50$ \\
\hline
\end{tabular}

a) $g$ = nozzle-to-substrate separation distance; ${ }^{b)} \mathrm{G}=$ green $\mathrm{PHOLED}, \mathrm{R}=$ red PHOLED; ${ }^{c}$ S-C = simple converging nozzle, $C-D=$ converging-diverging nozzle.
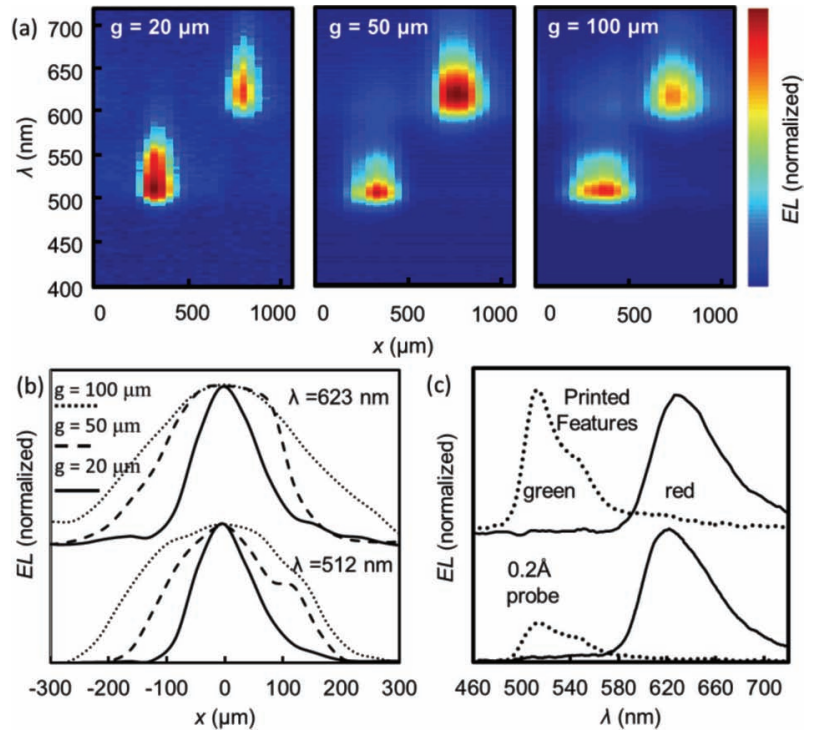

Figure 3. a) Spectrally resolved line scans of electroluminescence from an array of red and green devices. The scan direction along $x$ is orthogonal to the direction of printing. Wavelength $(\lambda)$ is along the abscissa, and the color indicates the relative intensity at each wavelength and scan step. Scans are shown for nozzle-to-substrate gaps of $g=20,50$, and $100 \mu \mathrm{m}$ The regions of emission become larger and have poorly defined borders for larger values of $g$. b) Normalized electroluminescent intensity as a function of distance from the center for emissive segments. The intensity profiles for the red segments are taken at $\lambda=623 \mathrm{~nm}$ and for the green segments at $\lambda=512 \mathrm{~nm}$. c) (Top) Spectrally resolved emission from the green (dots) and red (solid) segments of an array printed using a C-D nozzle at $g=20 \mu \mathrm{m}$. Data are normalized to the maximum spectral intensity of each feature. Note the absence of cross contamination between features. (Lower) Spectra from 0.2 A -thick RD-15 (solid) and $\operatorname{Irppy}_{3}$ (dots) films. Curves are normalized to the maximum intensity of the RD-15 signal.

Electroluminescent structures with a $0.2 \AA$ neat film of either green dopant Irppy 3 or red dopant RD-15 (Universal Display Corp. Ewing, NJ) deposited in place of the emissive layer of the OLED architecture used in this study (see Experimental Section) were fabricated to assess sensitivity of printed features to cross-contamination. Emission from RD-15 was roughly ten times the detection threshold of the line scanner, despite the very thin layer deposited. This implies that the overspray detection limit is $\approx 0.02 \AA$, or $1 / 500$ of a monolayer of the dopant. Since host-to-dopant energy transfer is negligible for films much thinner than a monolayer, a submonolayer film of dopant provides a useful gauge for the sensitivity of this technique for the presence of oversprayed materials. Contamination from RD-15 is more visible than Irppy $y_{3}$, and therefore more problematic. Emission intensity from a $0.2 \AA$-thick film of $\operatorname{Irppy}_{3}$ at its peak of $\lambda=512 \mathrm{~nm}$ is only $28 \%$ of that of RD-15 at $\lambda=623 \mathrm{~nm}$, as shown in the lower portion of Figure $3 \mathrm{c}$.

Red pixels are significantly wider than the green pixels at their bases (i.e., their width measured at $10 \%$ of the maximum intensity). Since both Irppy ${ }_{3}$ and RD-15 have comparable sublimation temperatures, this difference is due to the more intense emission from oversprayed RD-15 than from Irppy 3 . Red emission can be detected at $61 \mu \mathrm{m}$ beyond the FWHM on 

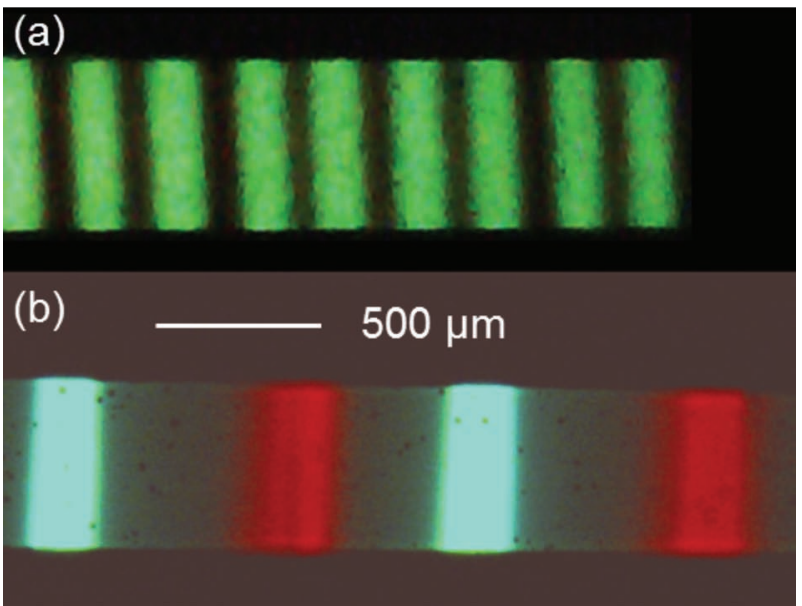

Figure 4. a) Photograph of emission from an array of green phosphorescent organic light-emitting devices printed using a simple converging nozzle at a nozzle-to-substrate distance of $g=20 \mu \mathrm{m}$. b) Array of red and green device segments printed using a converging-diverging nozzle at $g=20 \mu \mathrm{m}$.

each side of a feature printed using a C-D nozzle at $g=20$. No cross-contamination between red and green features printed on $500 \mu \mathrm{m}$ centers is detectible, as is evident from their emission spectra shown in the upper portion of Figure 3c. Green features printed at $\mathrm{g}=20$ have Commission Internationale de l'Éclairage (CIE) 1931 chromaticity ${ }^{[18]}$ co-ordinates $(0.27,0.63)$, which matches that of discrete Irppy $_{3}$ OLEDs. $^{[19]}$ Also, red devices have co-ordinates of $(0.66,0.33)$ that match the discrete value of $(0.66,0.32) .{ }^{[15]}$ Conversely, for $g=100 \mu \mathrm{m}$, red emission can be detected across the entire array. This contamination is sufficient to shift the CIE co-ordinates of a neighboring green segment to $(0.32,0.61)$. This spectrum corresponds to approximately $0.03 \AA$ of red dopant contamination, demonstrating that even a minute amount of RD-15 can adversely affect the performance of the green OLED. In contrast, the CIE co-ordinates of the red segment is unchanged for $g=100 \mu \mathrm{m}$, since transfer of triplet excitons from the red to green dopant is endothermic, and hence unlikely to occur.

Figure 4a shows an array of top emitting green pixels printed with the S-C nozzle array at $g=20 \mu \mathrm{m}$. The illuminated regions are approximately $130 \mu \mathrm{m}$ wide, corresponding to the full width at $10 \%$ of maximum intensity. An array of red and green electrophosphorescent stripes with alternating color subpixels by a C-D nozzle at $\mathrm{g}=20 \mu \mathrm{m}$ is shown in Figure 4b. Illuminated regions are 200 to $250 \mu \mathrm{m}$ in width, also corresponding to the measured width to $10 \%$ of maximum intensity for features printed by C-D nozzle. Note that in both cases, the emission intensity across the pixel width is uniform, indicating no adverse affects resulting from the thickness variations present in the feature.

Assuming that the emission intensity from red contamination in a green device varies linearly with concentration, an array of alternating $100 \mu \mathrm{m}$-wide red and green subpixels printed using C-D nozzles at $g=20 \mu \mathrm{m}$ require a center-tocenter spacing of at least $210 \mu \mathrm{m}$ to avoid noticeable spectral shifts (as determined by $2 \%$ changes in their CIE co-ordinates).
Higher feature densities can be obtained by reducing g. In Figure $5 \mathrm{a}$, we show a plot of the simulated deposition profile for a S-C nozzle for $g=10 \mu \mathrm{m}$, which is the minimum practical nozzle-to-substrate spacing when printing over large (e.g., Gen 8 ) substrate areas. Features must be spaced such that contamination beyond a detection threshold of $0.02 \AA$ is absent in the adjacent feature. Under this constraint, the minimum lateral subpixel spacing at $g=10 \mu \mathrm{m}$ is $70 \mu \mathrm{m}$ for $25 \mu \mathrm{m}$-wide features. Wider pixels can be printed in multiple passes without increasing the width of the border required between features. For instance, $50 \mu \mathrm{m}$-wide subpixels on $95 \mu \mathrm{m}$ centers can be printed in two passes.

To achieve an even closer pixel spacing, lateral spreading of contaminants can be reduced with integrated polymer or other
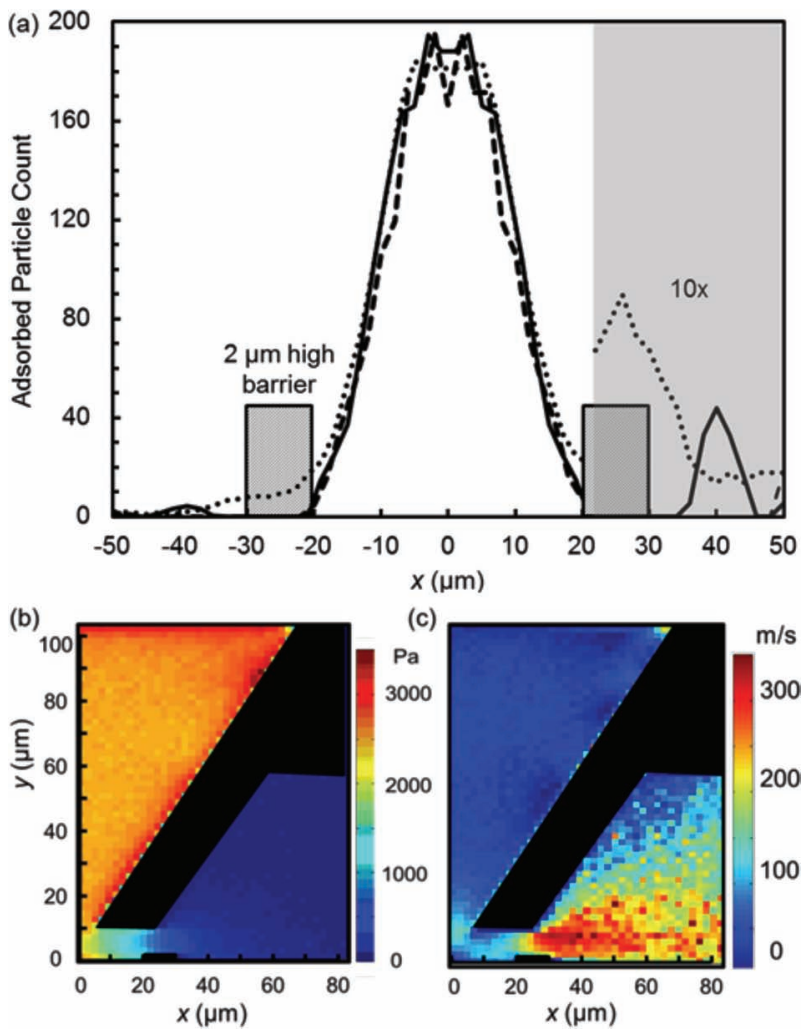

Figure 5. a) Modeled thicknesses of printed features as functions of distance from the nozzle centerline at $x=0$. The vertical axis indicates the number of organic molecules deposited on the substrate during a simulation run. The substrate is divided into $2 \mu \mathrm{m}$ bins for particle counting. Deposition is shown in the case of a simple converging (S-C) nozzle for a nozzle-to-substrate gap of $g=10 \mu \mathrm{m}$, both with (solid) and without (dotted line) $2 \mu \mathrm{m}$-high by $10 \mu \mathrm{m}$-wide barriers at the pixel edge. A deposition-free zone extending $10 \mu \mathrm{m}$ beyond the downstream edge of the barrier is apparent. The barrier can be combined with a nozzle featuring a tapered relief trench etched into its underside. The deposition profile produced by this combination is shown by the dashed line. To illustrate the small absolute differences in thicknesses downstream of the barrier more clearly, counts of deposited particles are multiplied 10x for profiles plotted in the shaded region on the right. Modeled (b) pressure and (c) velocity distributions are presented for an S-C nozzle with a relief trench etched into its underside. The barrier is visible on the lower surfaces of these plots. Organic material passing the barrier is deflected upward into the trench and clear of the substrate. 
easily deposited and patterned inert barrier structures (e.g. $\mathrm{SiO}_{2}$ ) surrounding the pixel active area, analogous to "wells" used for inkjet printing. ${ }^{[20]}$ Organic molecules that do not immediately adsorb on the cold substrate beneath the nozzle aperture move outward along a trajectory nearly parallel to the substrate plane. Even a relatively low barrier of $2 \mu \mathrm{m}$ significantly reduces downstream deposition by collecting stray organic vapor near the surface, as shown by the calculated profile for $\mathrm{g}=10 \mu \mathrm{m}$ in Figure 5a. Modeling indicates that no deposition occurs for $10 \mu \mathrm{m}$ past the downstream edge of the barrier. A small amount of material, deposits further downstream. If the deposited feature is a $20 \mathrm{~nm}$-thick emission layer for a PHOLED with $6 \%$ dopant, this simulated overspray corresponds to a neat dopant layer approximately $0.03 \AA$ thick, which is close to the threshold required to shift the CIE co-ordinates of a neighboring feature. The nozzle aperture must be in close proximity to the substrate for the barrier structure to be effective. For larger $g$, peripheral organic molecules approach the substrate at a steeper trajectory, shortening the shadow cast by the barrier and increasing overspray.

Carrier gas streams over the barriers, which in turn imparts vertical momentum to residual organic molecules in the flow. This organic material may deposit on the substrate if it scatters off the underside of the heated nozzle and is redirected downward. The effectiveness of barriers can, therefore, be enhanced by etching relief trenches into the downstream portion of the nozzle membrane. ${ }^{[13]}$ Such a nozzle cross section is shown in Figures $5 \mathrm{~b}$ and c, incorporating a $50 \mu \mathrm{m}$ deep relief trench with a sidewall parallel to the inner taper of the converging nozzle inlet. The combination of this nozzle geometry and the barriers eliminates virtually all stray deposition, as shown in the shaded portion of Figure 5a. There is virtually no deposition beyond the barrier in the simulated structure, implying that overspray of dopant beyond the barrier would be below the threshold for cross-contamination of adjacent pixels. Subpixels $40 \mu \mathrm{m}$ wide can therefore be printed on $50 \mu \mathrm{m}$ centers by a micronozzle array with relief trenches using $2 \mu \mathrm{m}$-high, $10 \mu \mathrm{m}$-wide substrate barriers. A full-color pixel pitch of $150 \mu \mathrm{m}$ can thus be achieved, which is comparable to that achieved with thin metal shadow masks. ${ }^{[21]}$ Further optimization may lead to even closer pixel spacing. Other nozzle and barrier shapes (e.g. undercut barriers that trap organic material without re-directing free material upwards), as well as strategic relative placement of red, green and blue subpixels to minimize color changes in the presence of overspray can result in even higher display image resolution using this printing method.

A fraction of the organic material that flows past the barrier structure will result in contamination of neighboring areas. For the case of S-C nozzles, $12 \%$ of organic material leaves the $80 \mu \mathrm{m}$-wide simulation volume at $\mathrm{g}=50 \mu \mathrm{m}$, but $<0.1 \%$ escapes for $g=10 \mu \mathrm{m}$. Small values of $g$ are, therefore, crucial to avoid cross contamination. For example, $0.1 \%$ of the material required to grow a $200 \AA$-thick emissive layer corresponds to $\approx 1 / 1000$ of a monolayer of deposit, which is below the detection threshold.

The printed device performance was similar to that obtained for devices with the same layer structure grown entirely by vacuum thermal evaporation (VTE). Top-emitting green PHOLEDs grown by OVJP have peak external quantum efficiencies (EQE) of $4.9 \pm 0.5 \%$, compared with $5.4 \pm 0.7 \%$ for analogous, VTE-grown devices. While this EQE is only half that expected for optimized Irppy ${ }_{3}$ PHOLEDs, ${ }^{[19]}$ the structure used is designed to evaluate the presence of overspray and not for high efficiency. In particular, the top emitting architecture has $\mathrm{a} \approx 49 \%$ lower outcoupling efficiency than a bottom emitting device, ${ }^{[22]}$ accounting for the discrepancy. It has previously been shown that high efficiency PHOLEDs can be achieved by this printing method. ${ }^{[13]}$

In conclusion, multicolor PHOLEDs were printed by OVJP in a single pass using micronozzle arrays. The red dopant, RD-15, was shown to be a highly sensitive indicator of contamination between adjacent pixels, with a detection limit of only 1/500 of a monolayer. Two nozzle geometries were evaluated: S-C nozzles produced narrower features with sharply defined boundaries compared with C-D nozzles due to a lower carrier gas velocity underneath the S-C nozzle aperture. Simulations indicate that an array of $40 \mu \mathrm{m}$-wide subpixels can be printed on $50 \mu \mathrm{m}$ centers without detectable cross-contamination using $2 \mu \mathrm{m}$ high barrier structures between printed features, a nozzleto-substrate distance of $10 \mu \mathrm{m}$, and an array of S-C nozzles with $20 \mu \mathrm{m}$-wide apertures and relief trenches. This implies that color displays with a full-color pixel pitch of $150 \mu \mathrm{m}$ can be fabricated using OVJP. Printed Irppy ${ }_{3}$ devices fabricated using a micronozzle array performed comparably to analogous devices grown entirely by VTE.

\section{Experimental Section}

An array of $\mathrm{Si}$ micronozzles was fabricated using standard microelectromechanical systems (MEMS) processing procedures. ${ }^{[14]}$ The $100 \mu \mathrm{m}$-thick device layer of a $<100>$ Si-on-insulator (SOI) wafer with a $1 \mu \mathrm{m}$-thick buried oxide layer is etched with $\mathrm{KOH}$ to form the inner surface of the nozzle membrane. ${ }^{[14]}$ The device layer is then anodically bonded to a borosilicate glass wafer. ${ }^{[23]}$ The Si handle layer is removed by deep reactive ion etching, using the buried oxide as an etch stop. The oxide is then patterned for use as a hard mask. The outer side of the membrane is etched in an ethylenediamine pyrocatechol bath to produce features with sloped sidewalls along $\langle 111\rangle$ Si. ${ }^{[24]}$ Following nozzle formation, the wafers were diced into $(1 \mathrm{~cm})^{2}$ sections. The nozzle membranes were released from their glass handle by etching in HF.

Nozzle membranes were soldered to a Kovar plate containing $2 \mathrm{~mm}$-wide channels used to mix organic vapors from different source cells and transport them to the nozzles. The channel plate contains two sets of channels, one for each color. Following lapping and polishing ${ }^{[13]}$ of the plate, it and the Si nozzle array surfaces are coated with thermally evaporated layers ${ }^{[25]}$ of $20 \mathrm{~nm}$-thick $\mathrm{Cr}$ and $30 \mathrm{~nm}$-thick Pt, followed by two sequential depositions of a $266 \mathrm{~nm}$-thick Au and $160 \mathrm{~nm}$-thick Ge layer, and capped with a $133 \mathrm{~nm}$-thick Au layer. This layer stack was capped with a $133 \mathrm{~nm}$-thick Au layer. Thicknesses are chosen to produce a Au-Ge eutectic mixture when heated. Membranes were joined to the channel plate under high vacuum at $400{ }^{\circ} \mathrm{C}$ and $1-3 \mathrm{MPa}$ platen pressure for $1 \mathrm{hr}$.

The print head, consisting of the channel plate, resistive heaters, and nozzle membrane, is sealed to a manifold containing multiple organic vapor sources using Kalrez O-rings. Each vapor source consists of a vented Pyrex vial containing the organic source material, thermocouple, heater, and mass flow controller as described previously. ${ }^{[13]}$ Separate temperature control of each vial allows for deposition of materials with different evaporation temperatures. The print head is located in a vacuum chamber with a base pressure $<10^{-5}$ Torr. The chamber is connected to a glove box filled with ultrahigh purity $\mathrm{N}_{2}$, where substrates can be transferred to a thermal evaporation system without exposure to 
atmosphere. Substrates are placed on a chilled holder that is translated by a precision, 4-axis $(x, y, z, \theta)$ motorized stage. Vertical position is measured using a fiber optic displacement sensor.

Top emitting PHOLEDs were grown on a $\mathrm{Si}$ substrate with a $300 \mathrm{~nm}$-thick Al and $20 \mathrm{~nm}$-thick Pt anode deposited over the entire surface by VTE in a chamber with a base pressure of $\approx 10^{-7}$ torr. A $50 \mathrm{~nm}$-thick hole-transport layer of $\mathrm{N}, \mathrm{N}^{\prime}$-di(1-naphthyl)-N, $\mathrm{N}^{\prime}$ diphenylbenzidine and a $10 \mathrm{~nm}$-thick fac-tris(1-phenylpyrazolato, $\mathrm{N}, \mathrm{C2}^{\prime}$ ) iridium(III) electron blocking layer were deposited over the anode. The light emitting layer consists of OVJP-printed stripes of the host material, CBP mixed with one of two phosphorescent dopants, either the green emitter $\operatorname{Ir}(\mathrm{ppy})_{3}$, or the red emitter RD-15. The overall deposition rate was controlled via the temperature of the source cells, while the doping ratio was controlled by the fraction of the total carrier gas flow sent through the dopant source. ${ }^{[13]}$ The doping ratio was calibrated prior to deposition using photoluminescence spectroscopy. That is, the doping ratio employed corresponds to the minimum guest concentration leading to complete quenching of the CBP host emission. Hence, a dopant ratio of $6 \pm 3$ vol\% for $\operatorname{Irppy}_{3}{ }^{[19]}$ and $10 \pm 3$ vol\% for RD-15, ${ }^{[15]}$ were used in these experiments. The thickness of the emissive stripes was approximately $20 \mathrm{~nm}$, measured using stylus profilometry. The Irppy $_{3}$ source was heated to $330{ }^{\circ} \mathrm{C}, \mathrm{CBP}$ sources were between $300{ }^{\circ} \mathrm{C}$ and $340{ }^{\circ} \mathrm{C}$, and the RD-15 source was at $350{ }^{\circ} \mathrm{C}$. Ultrapure $\mathrm{N}_{2}$ carrier gas was fed into each material source at a flow rate of from 0.1 to $1 \mathrm{sccm}$, generating a pressure of between 3 and 10 torr within the source. The print head temperature was $350{ }^{\circ} \mathrm{C}$ to prevent downstream organic condensation, and the substrate stage was chilled to between 0 and $-20^{\circ} \mathrm{C}$. A gap, g, of between $20 \mu \mathrm{m}$ and $100 \mu \mathrm{m}$ was maintained between the nozzle outlets and the substrate. The substrate was translated relative to the nozzle array at between 8 and $12 \mathrm{~mm} / \mathrm{s}$ along the printing axis. Both red and green stripes were simultaneously printed in a single pass.

Following printing, a $20 \mathrm{~nm}$-thick 4,7-diphenyl-1,10-phenanthroline hole blocking layer and a $25 \mathrm{~nm} \mathrm{Al} \mathrm{(III)} \mathrm{tris(8-hydroxyquinolate)} \mathrm{electron-}$ transport layer are blanket-deposited over the substrate by VTE. Devices are then capped with a semi-transparent, $1 \mathrm{~nm}$-thick LiF, $5 \mathrm{~nm}$-thick $\mathrm{Al}$, and $15 \mathrm{~nm}$-thick $\mathrm{Ag}$ cathode that is patterned using a striped shadow mask oriented perpendicular to the striped emitting layer. ${ }^{[22]}$ Electroluminescence of the printed patterns was measured using a motorized micrometer line scanner. An optical fiber placed at the image plane of a 10x objective coupled light into a spectrometer, yielding a spatial resolution of $40 \mu \mathrm{m}$.

Deposition was modeled using the non-time counting direct simulation Monte Carlo method. ${ }^{[7]}$ The simulated domain was divided into $2 \mu \mathrm{m}$ square bins for purposes of collision selection and calculation of output values. Hard sphere collisions were assumed to account for intermolecular interactions. Model organic molecules have a mass of $500 \mathrm{~g} \mathrm{~mol}^{-1}$ and a diameter of $1 \mathrm{~nm}$. The surfaces of the nozzle act as diffuse reflectors for all particles. The plane of the substrate and raised barrier structures act as getters for organic material and diffusely reflect carrier gas. Organic tracer molecules were seeded into the carrier gas at a ratio of 1:1000 such that their presence does not significantly affect the dynamical properties of the gas flow.

Received: October 23, 2012

Revised: November 29, 2012 Published online: January 20, 2013
[1] B. Young, Inf. Disp. 2009, 25, 14-17.

[2] C. Adachi, M. A. Baldo, M. E. Thompson, S. R. Forrest, J. Appl. Phys. 2001, 90, 5048-5051.

[3] A. Nathan, G. R. Chaji, S. J. Ashtiani, J. Disp. Technol. 2005, 1, 267-277.

[4] H.-N. Lee, J. Kyung, M.-C. Sung, D. Y. Kim, S. K. Kang, S.-J. Kim, C. N. Kim, H.-G. Kim, S.-T. Kim, J. Soc. Inf. Disp. 2008, 16, 265-272.

[5] J.-H. Lee, D. N. Liu, S.-T. Wu, Introduction to Flat Panel Displays, Wiley, New York 2009

[6] P. F. Tian, V. Bulovic, P. E. Burrows, G. Gu, S. R. Forrest, T. X. Zhou, J. Vac. Sci. Technol. A 1999, 17, 2975-2981.

[7] Y. Fukuda, T. Watanabe, T. Wakimoto, S. Miyaguchi, M. Tsuchida, Synth. Met. 2000, 111, 1-6.

[8] H. Kobayashi, S. Kanbe, S. Seki, H. Kigchi, M. Kimura, I. Yudasaka, S. Miyashita, T. Shimoda, C. R. Towns, J. H. Burroughes, R. H. Friend, Synth. Met. 2000, 111, 125-128.

[9] M. B. Wolk, J. Baetzold, E. Bellmann, T. R. Hoffend, S. Lamansky, Y. B. Li, R. R. Roberts, V. Savvateev, J. S. Staral, W. A. Tolbert, in Organic Light-Emitting Materials and Devices Viii (Eds.: Z. H. Kafafi, P. A. Lane), SPIE-Int Soc Optical Engineering, Bellingham, WA, USA 2004, pp. 12-23.

[10] V. Leblanc, J. Chen, P. Mardilovich, V. Bulovic, M. A. Schmidt, in Solid-State Sensors, Actuators and Microsystems Conference, 2007, pp. 121-124.

[11] David K. Flattery, C. R. Fincher, D. L. LeCloux, M. B. O'Regan, J. S. Richard, Inf. Disp. 2011, 27, 8-13.

[12] M. Shtein, P. Peumans, J. B. Benziger, S. R. Forrest, Adv. Mater. 2004, 16, 1615-1620.

[13] G. J. McGraw, S. R. Forrest, J. Appl. Phys. 2012, 111, 043501-043501-8.

[14] G. J. McGraw, D. L. Peters, S. R. Forrest, Appl. Phys. Lett. 2011, 98, 013302-013302-3.

[15] M. S. Arnold, G. J. McGraw, S. R. Forrest, R. R. Lunt, Appl. Phys. Lett. 2008, 92, 053301-053301-3.

[16] W. F. Louisos, A. A. Alexeenko, D. L. Hitt, A. Zilic, Int. J. Manuf. Res. 2008, 3, 80 .

[17] G. A. Bird, Molecular Gas Dynamics and the Direct Simulation of Gas Flows, Oxford University Press, New York 1994.

[18] T. Smith, J. Guild, Trans. Opt. Soc. 1931, 33, 73-134.

[19] M. A. Baldo, S. Lamansky, P. E. Burrows, M. E. Thompson, S. R. Forrest, Appl. Phys. Lett. 1999, 75, 4-6.

[20] J. Steiger, S. Heun, N. Tallant, J. Imaging Sci. Technol. 2003, 47, 473-478.

[21] J.-J. Lih, C.-I. Chao, C.-C. Lee, J. Soc. Inf. Disp. 2007, 15, 3-7.

[22] L. S. Hung, C. W. Tang, M. G. Mason, P. Raychaudhuri, J. Madathil, Appl. Phys. Lett. 2001, 78, 544-546.

[23] K. M. Knowles, A. T. J. van Helvoort, Int. Mater. Rev. 2006, 51, 273-311.

[24] A. Reisman, M. Berkenblit, S. A. Chan, F. B. Kaufman, D. C. Green, J. Electrochem. Soc. 1979, 126, 1406-1415.

[25] S. Lani, A. Bosseboeuf, B. Belier, C. Clerc, C. Gousset, J. Aubert, Microsyst. Technol. 2006, 12, 1021-1025. 